\title{
The Effect of High Power at Microwave Frequencies on the Linearity of Non-Polar Dielectrics in Space RF component
}

\author{
Tawfik Elsayed Khattab \\ Department of Electrical Engineering, Engineering College, King Khalid University, Abha, Saudi Arabia \\ Email: tkhtab@kku.edu.sa
}

Received 2 February 2015; accepted 21 February 2015; published 28 February 2015

Copyright (C) 2015 by author and Scientific Research Publishing Inc. This work is licensed under the Creative Commons Attribution International License (CC BY). http://creativecommons.org/licenses/by/4.0/

(c) (i) Open Access

\begin{abstract}
In satellite communication systems, there are high power multichannel transmitters and wideband receivers that have shared RF antenna transmission lines because of: 1) large power level difference between the transmitted and received signal; 2) limited frequency channels. The harmonics and Passive Intermodulation (PIM) Interference will be generated due to passive non-linearities in the high power transmission path. This can be a serious problem. This paper describes how to determine the signal levels and dominant mechanisms that are associated with non-linear dielectric behavior in this context. A novel measurement system for testing dielectric samples is described and measurement results are provided for commonly used microwave dielectrics.
\end{abstract}

\section{Keywords}

Passive Intermodulation, PIM, Nonlinear, Polar Dielectric, Harmonics, Cavity Resonator

\section{Introduction}

Passive Intermodulation, PIM, is a form of intermodulation distortion that can occur even when no active components are present. PIM can occur in a variety of areas from coaxial connectors to cables, even rusty bolts or any joint where dissimilar metals occur [1]. Although harmonics generation is a ubiquities phenomenon, very little is known of the underlying causative mechanisms, relating to either conductors or dielectrics. There are a large number of candidate mechanisms and it is likely that several may contribute at any given site of occurrence. There currently exists no data on the levels of the harmonics to be expected from commonly used dielectric materials nor have any studies identified the dominant mechanisms. As greater demand is placed under system performance, it is becoming increasingly difficult to provide adequate harmonic generation immunity in the 
absence of data. The purpose of this work is to provide an improved understanding of causes and effects of the non-linearity of dielectrics leading to improvements in RF system design. The specific objectives are as follows:

- To design and develop a measurement system whereby the non-linear behavior of dielectric materials under test can be measured.

- To characterize the non-linear behavior of dielectrics in common use in microwave RF engineering, such as PTFE and Polystyrene.

\section{The Measurement System}

The measurement system is a new design of harmonic detector, implemented in coaxial components. The system is designed for $1.56 \mathrm{GHz}$ to detect the $3^{\text {rd }}$ harmonic at $4.68 \mathrm{GHz}$, due to any significant non-linear behavior of dielectric test samples. Dielectric samples are tested in a coaxial cavity, where they are exposed to a high uniform electric field. Because the level of system residual $3^{\text {rd }}$ harmonic must be kept to minimum, all components are in-house designs in which metal-to-metal junctions are absent from critical sections in the current path. Important features are the use of contactless cable entry for critical components and fully demountable contactless coaxial connectors on test cavity.

\subsection{System Configuration}

A block diagram of the $3^{\text {rd }}$ harmonic measurement setup is given in Figure 1. A source signal of $1.56 \mathrm{GHz}$, originated from a phase locked oscillator, is amplified by a separate power amplifier to give an output power up to $49 \mathrm{dBm}$ (80 Watts).

The source signal passes through a diplexer using two hybrids and two low pass filters and hence to the input port of the test cavity. The low pass filters pass only the signal at the source frequency and attenuate any harmonics which may be generated in the oscillator or in the power amplifier. The diplexer provides a test signal to the cavity and couples the $3^{\text {rd }}$ harmonic which may be generated in the test chamber due to any non-linear behavior of the sample under test.

This consists of a high pass filter which attenuates the source frequency and passes the $3^{\text {rd }}$ harmonic to the input port of the analyzer. Source signal power is absorbed in a quiet load. This consists of two semi rigid coaxial cables, UT250 with length $45 \mathrm{~m}$, followed by UT-141 with length $20 \mathrm{~m}$, terminated by a lumped load. The total one way attenuation for the quiet load is $30 \mathrm{~dB}$.

The test chamber is a tunable quarter-wave coaxial cavity resonator which satisfies the following requirements:

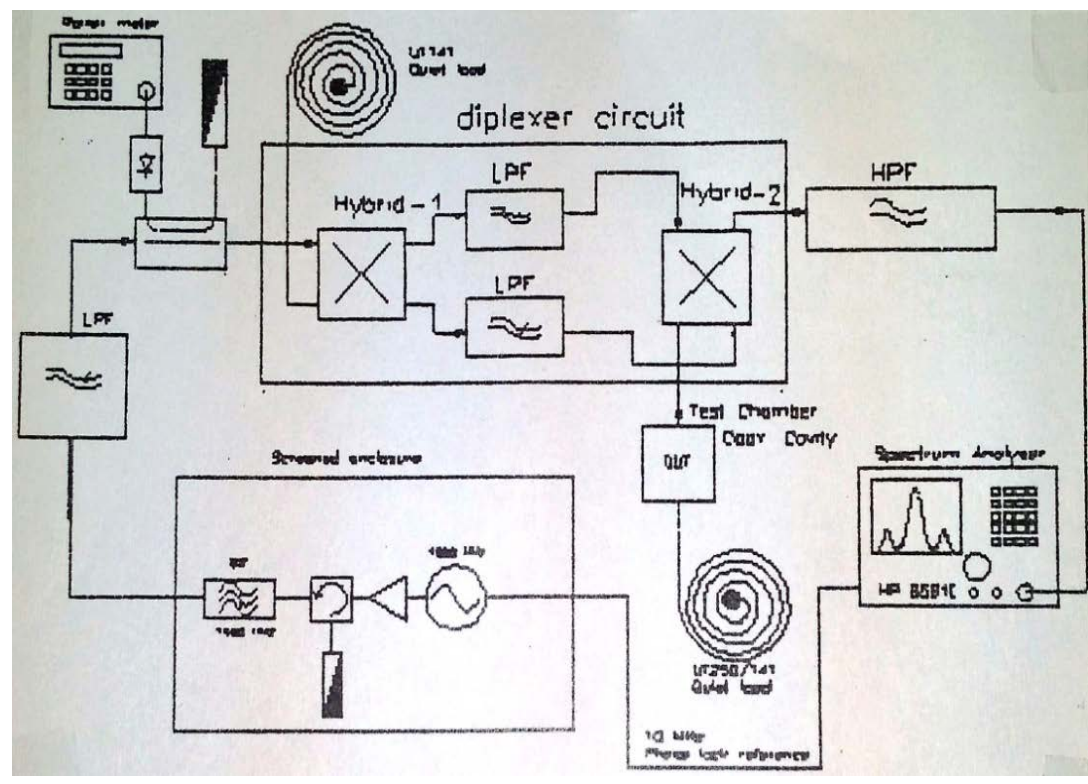

Figure 1. The Measurement system block diagram. 
- Flexibility to accommodate and exchange test samples.

- The test samples can be tested under controlled conditions.

- Good matching between input and output ports. This is important since the system residual can be degraded by current standing waves.

- Good linearity is essential. If the test chamber construction includes non-linear materials or vulnerable junctions, consequently the system residual intermodulation will be increased, and this will mask the intermodulation signal produced by the test samples, which affect the dynamic range of the measurement system.

- A high uniform field at the position of the test sample.

The test cavity is machined in brass and is shown in Figure 2. It incorporates contactless connection at cable entry, one piece body/resonator constriction and a contactless plunger to control the gap capacitance. Past experience in the design of low passive intermodulation coaxial components has shown that these features are important in achieving a low residual. The test field is determined from measurement of the cavity $\mathrm{Q}$ factor and the source signal power, given the cavity geometry. The cavity loaded Q is in the region of 130 .

\subsection{The System Performance}

The electrical performance of the system has been tested using network analyzer. Figure 3 shows the transmission of the source signal and absolute attenuation for the harmonics at the output of the test chamber. Figure 4 shows the absolute attenuation for the source signal and transmission of the $3^{\text {rd }}$ harmonic at the output of the high pass filter.

The system noise flour is $-125 \mathrm{dBm}$ and the $3^{\text {rd }}$ order residual is $-115 \mathrm{dBm}$ at a source signal power of $32 \mathrm{~W}$ and increases at $3 \mathrm{~dB}$ rate. It should be noted that these figures should be related to the field in the test cavity, rather than the source power, as in a conventional two tone system.

\section{Dielectric Non-Linear Mechanisms}

The candidate non-linear mechanisms which may reasonably be suspected as significant causes of dielectric harmonics generation are considered to be non-linear permittivity, electrostriction and temperature coefficient of

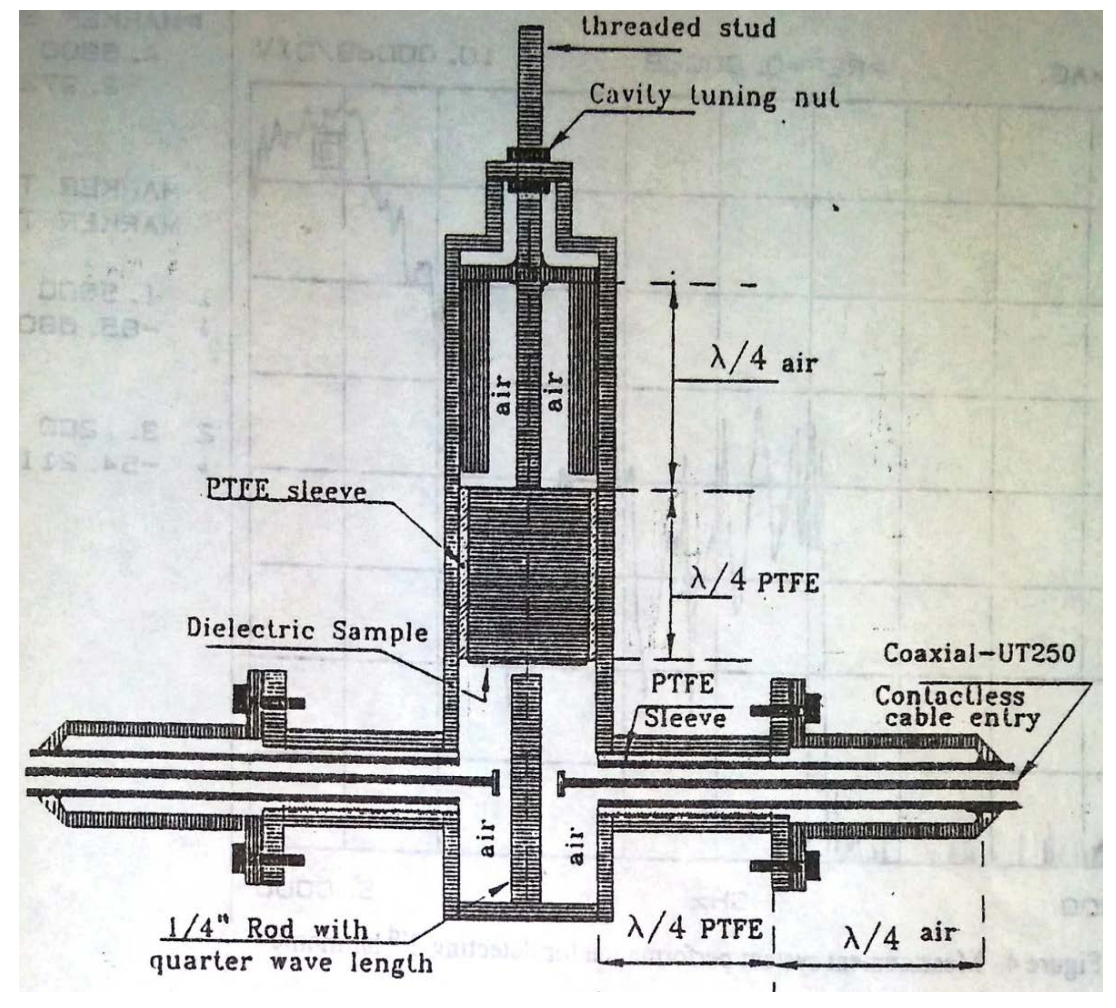

Figure 2. Test cavity. 


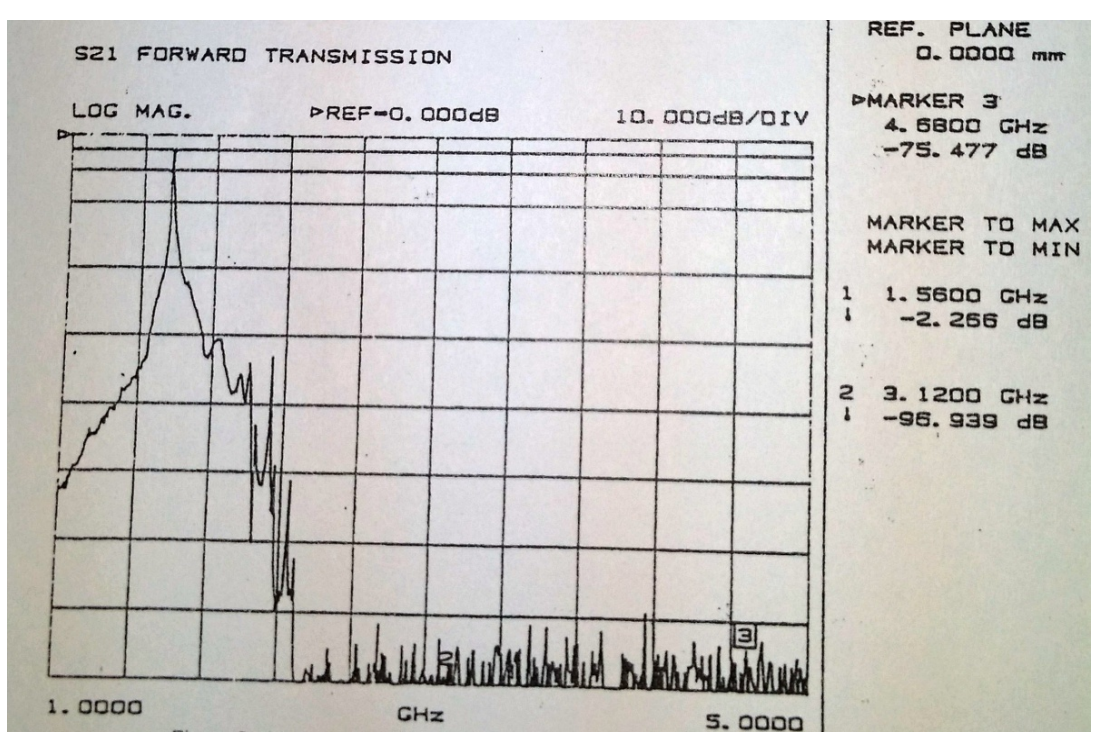

Figure 3. Measurement system performance in transmission mode.

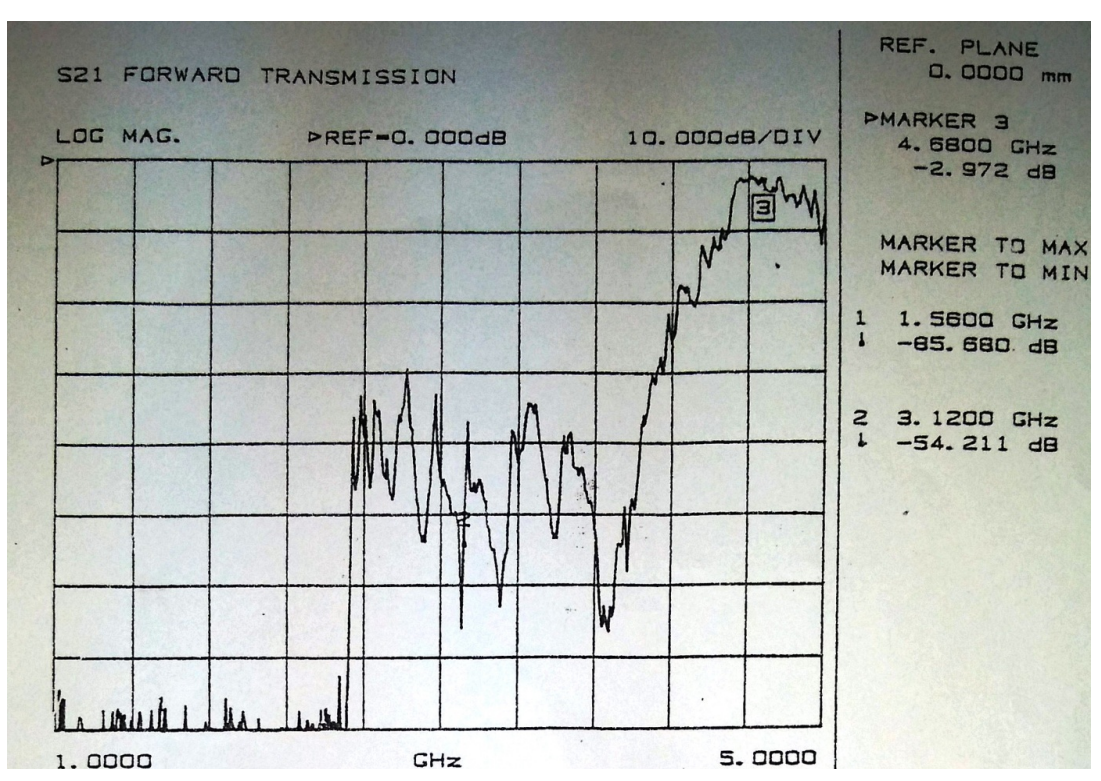

Figure 4. Measurement system performance for detecting $3^{\text {rd }}$ Harmonic.

permittivity. Many potential causes of harmonics generation arise from intersection of two or more linear mechanisms which may give rise to multi-order non-linear behavior. This results in a bewildering number of possible combinations. An outline theoretical analysis is provided for candidate mechanisms and their relative importance is estimated based on theoretical considerations.

\subsection{Non-Linear Permittivity}

The linear relationship between Polarization, $P$, and electric field strength, $E$, in dielectrics is expressed by:

$$
P=\varepsilon_{0} \gamma E
$$

where $\gamma$ is the susceptibility and $\varepsilon_{0}$ is the permittivity of free space [2]-[4].

The value for the field at which deviation from linearity becomes apparent is given as around $1000 \mathrm{v} / \mathrm{mm}$ [5]. This implies high, but not unreasonable, current densities in the dielectric at microwave frequencies. The nonlinear polarization may be modeled as power series of susceptibility terms. Only odd powers will be present for 
dielectrics which do not exhibit spontaneous polarization, since a polarity reversal in the electric field reverses the direction of polarization but does not alter its intensity. The $1^{\text {st }}$ non-linear term to become significant is cubic in the electric field:

$$
P=\varepsilon\left(\gamma E+\aleph E^{3}\right)
$$

where $\aleph$ is the $3^{\text {rd }}$ order susceptibility coefficient. This gives rise to a current term that is also proportional to the cube of the electric field and the $3^{\text {rd }}$ order intermodulation products will be generated. Alternatively, if this behavior is expressed as a non-linear relative permittivity $\varepsilon_{r}$, so that $P=\varepsilon_{0}\left(\varepsilon_{r}-1\right) E$, then the $1^{\text {st }}$ non-linear term may be described as quadratic (since $\varepsilon_{r}=\gamma+\aleph E^{2}$ ) and only even terms exist.

For dielectric materials which exhibit spontaneous polarization, deviations from linear behavior may become apparent at much lower field strengths and both odd and even powers are possible. Stauss G.H. [5] dismiss non-linear permittivity as a source of harmonics generation in dielectrics on the grounds that it is less significant than the indirect modulation of permittivity by means of electrostriction it should be noted; however, that the examples given are good non-polar dielectrics such as Teflon which are selected for their desirable properties as insulators at radio frequencies and are of high purity. Thus only electronic polarization contributes to the relative permittivity and these materials are not representative of dielectrics in general. Ionization loss occurs in solids which contain trapped gas. It only occurs when the electric field strength exceeds a critical value, above which the loss tangent rises rapidly with any increase in the field. Thus the imaginary component of the complex permittivity becomes non-linear and intermodulation components could appear in the conduction current. The increased power dissipation can also result in significant greater heating of the dielectric.

\subsection{Temperature Coefficient of Permittivity}

The permittivity of dielectrics which only exhibits electronic polarization typically have small negative temperature coefficient of around $0.01 \% \mathrm{C}^{-1}$. This is primarily due to the reduction of density as the material expands. The same effect also tends to reduce atomic polarization; but for many materials this is more than compensated by the reduced inter-atomic forces in a less dense medium. This results in a positive temperature coefficient of typically $0.01 \% \mathrm{C}^{-1}$. There are exceptions to this: Titanium dioxide, for example, has negative temperature coefficient of permittivity at room temperature. As these forms of polarization result in very small dielectric losses at microwave frequencies, they do not contribute significantly to loss tangent [6]-[8].

Orientational polarization and ionic relaxation polarization also generally make a positive contribution to the temperature coefficient permittivity. In some materials, orientational polarization that can exhibit much larger positive and negative temperature coefficients over a certain ranges is often associated with the melting point, but may begin at lower temperature while the material is still a solid. Ionic relaxation polarization exhibits an approximately exponential increase in the loss tangent with temperature. For orientation polarization, lose tangent typically rises to a maximum (at a temperature which depends on the frequency) and thin falls, before rising again as the increase in conductivity with temperature becomes significant.

Interfacial polarization is likely to be strongly influenced by temperature since it relies on the presence of free charges, but the nature of this effect will depend on the particular materials involved. In dielectrics which do not exhibit interfacial polarization, (typically exponential) increase in carrier density and conductivity with temperature is responsible for positive contribution to the overall temperature coefficient of the loss tangent. This is more significant for those materials which are poor insulators and at high temperatures. It has less effect on the loss tangent at higher frequencies, where a greater proportion of the current is conveyed by polarization mechanisms.

\subsection{Electrostriction}

Electrostriction is the volume change due to the variation of energy density in a dielectric under RF excitation. The change in volume causes a quadratic dependence of permittivity upon the electric field. Resulting permittivity variations at the fundamental and harmonics of the input frequency modulate the primary fields leading to the generation of harmonics.

If the high field polarization is non-linear in the field strength, the dependence of the dielectric displacement, $D$, on the field strength will also be non-linear [9]. 


$$
D=\varepsilon_{0} E+P=\varepsilon_{0} E+\varepsilon_{0}\left(\gamma E+\aleph E^{3}\right)
$$

When measurements of the non-linear effects are made by superposing a low intensity alternating field on static field of high intensity, the measurement results in the field dependent incremental dielectric permittivity $\varepsilon_{E}$ which is given by:

$$
\varepsilon_{E}=\frac{\partial D}{\partial E}=\varepsilon+3 \varepsilon_{0} \aleph E^{2}
$$

where $\varepsilon=\varepsilon_{0} \varepsilon_{r}$ and $\varepsilon_{r}=(1+\aleph)$, hence

$$
\frac{\Delta \varepsilon}{E^{2}}=\frac{\varepsilon E-\varepsilon}{E^{2}}=3 \varepsilon_{0} \aleph
$$

The non-linear effect is then characterized by the quantity $\frac{\Delta \varepsilon}{E^{2}}$ resonator rod and the top wall of the cavity as shown in Figure 1. Samples consist of $1 \mathrm{~mm}$ thick discs, lightly held in place, i.e. under constant pressure (p). The variation of the dielectric permittivity is given by:

$$
\varepsilon_{E}=\left(\frac{\mathrm{d} D}{\mathrm{~d} E}\right)_{T, d}+\left(\frac{\mathrm{d} D}{\mathrm{~d} d}\right)_{T, E}\left(\frac{\mathrm{d} d}{\mathrm{~d} E}\right)_{T, P}
$$

where, $T, d$ denote to the temperature and density of the sample under test. The first term of Equation (6) is the contribution to $\varepsilon_{E}$ due to the electrostriction; denoting this term by $\Delta \varepsilon_{e}$ [5].

$$
\Delta \varepsilon_{e}=\left(\frac{\mathrm{d} D}{\mathrm{~d} E}\right)_{T, d}=E^{2} \frac{1}{K} d^{2}\left(\frac{\partial \varepsilon}{\partial p}\right)_{T}
$$

Thus, the magnitude of the contribution to dielectric permittivity due to electrostriction can be calculated from the bulk modulus $(k)$ and the dependence of the permittivity on either the density or the pressure.

Intermodulation powers developed by this mechanism vary in inverse proportion to the square of the bulk modulus of the dielectric. The modulus, $k$, is related to the elasticity modulus and Poission's ratio by the following equation [10].

Where $E_{s}=$ elasticity modules, and $v=$ Poission's ratio. There is an associated thermal effect so that the volume change can also result in temperature variation. Electrostriction is expected to be the principal source of non-linearity in good non-polar dielectrics such as PTFE and cross-linked polystyrene because the bulk modules of these materials are very small.

\section{Results Analysis and Discussion}

Any variation in dielectric properties with the applied field will serve to modulate in coming signal currents. The $3^{\text {rd }}$ harmonic intermodulation level generated due to the non-linear mechanisms of the dielectric sample under test, and excited by a uniform field that can be derived based on power series as follows [11].

$$
D=\varepsilon_{0} \varepsilon_{r}\left(E+\alpha E^{3}+\beta E^{5}+\cdots\right)
$$

where $\alpha, \beta$ are coefficients of non-linear dielectric behavior.

$$
\begin{aligned}
& E=E_{0} \sin \omega t \\
& i= \varepsilon_{0} \varepsilon_{r} \frac{\partial}{\partial t}\left(E_{0} \sin \omega t+\alpha\left(E_{0} \sin \omega t\right)^{3}+\beta\left(E_{0} \sin \omega t\right)^{5}+\cdots\right) \\
&= \varepsilon_{0} \varepsilon_{r} \omega\left[\left(E_{0}+\frac{3}{4} \alpha E_{0}^{3}+\frac{5}{8} \beta E_{0}^{5}+\cdots\right) \cos \omega t\right. \\
&\left.+\left(-\frac{3}{4} \alpha E_{0}^{3}-\frac{5}{16} \beta E_{0}^{5}+\cdots\right) \cos 3 \omega t+\left(\frac{5}{16} \beta E_{0}^{5}+\cdots\right) \cos 5 \omega t+\cdots\right]
\end{aligned}
$$

Hence the $3^{\text {rd }}$ harmonic transfer function is given by: 


$$
I M_{H 3}=20 \log \left[\frac{-\frac{3}{4} \alpha E_{0}-\frac{5}{16} \beta E_{0}+\cdots}{E_{0}+\frac{3}{4} \alpha E_{0}+\frac{5}{8} \beta E_{0}+\cdots}\right]=20 \log \left(\frac{3}{4} \alpha E_{0}^{2}\right)
$$

The value of $\alpha$ determines the level of non-linearity of each dielectric material and will vary from one material to another according to the operative mechanism. The main contribution of this paper is the experimental results shown in Figure 5 and Table 1, which show non-linearity become significant in Nylon-66 at $700 \mathrm{~V} / \mathrm{mm}$ and polythene at $2000 \mathrm{~V} / \mathrm{mm}$ respectively. Hence, non-linear permittivity is the likely mechanism. The polystyrene shows a much better performance and the most linear material is PTFE. Hence, electrostriction is expected to be the main source of non-linearity. Good non-polar materials (such as PTFE, $\operatorname{AL}\left(\mathrm{AL}_{2} \mathrm{O}_{2}\right)$ ) need much higher powers to excite significant non-linearity.

\section{Conclusion}

These results demonstrate that care is needed in the use of dielectrics in any high field environment where $3^{\text {rd }}$ harmonic must kept to minimum. No measurements have been made on futile loaded materials, but these can be expected to demonstrate significant non-linearity due to non-linear permittivity. It must also be appreciated that any trace of moisture will cause similar behavior. This can be due to moisture absorption, which occurs to a varying extent, in most engineering dielectrics. Sample impurity is a farther factor and can cause significant degradation particularly where good performance is important.

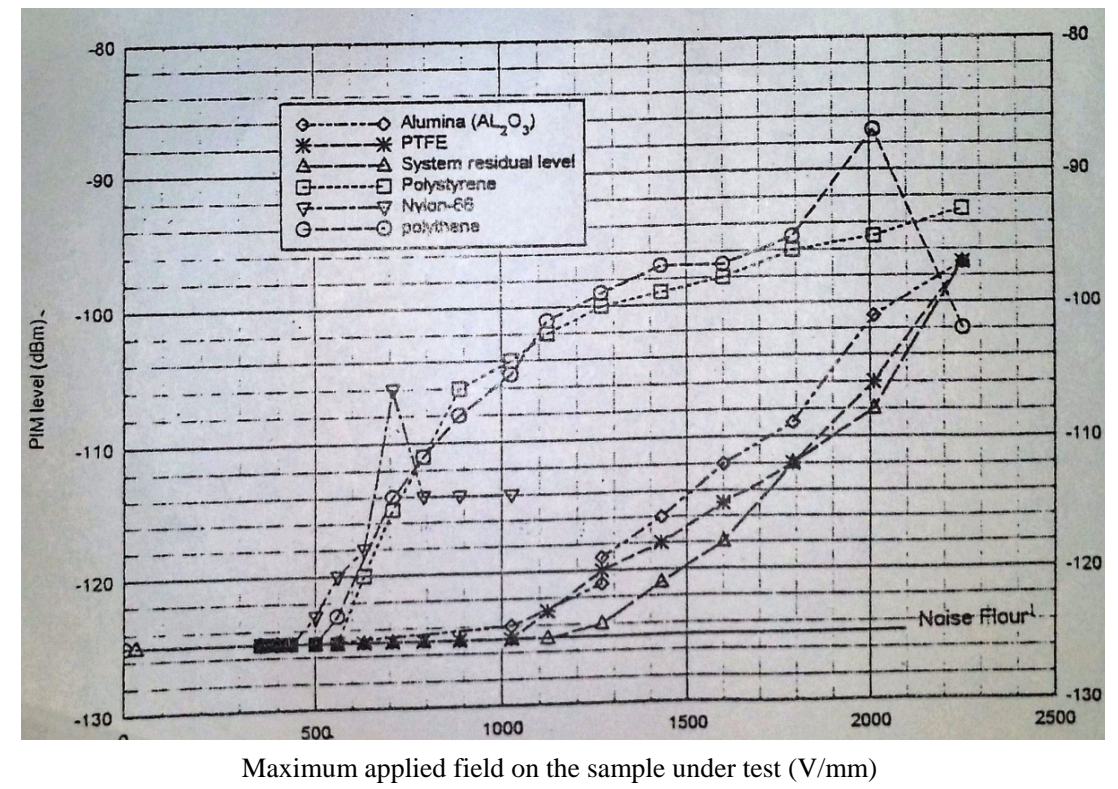

Figure 5. Measurement results.

Table 1. $3^{\text {rd }}$ harmonic level for the measured dielectric samples.

\begin{tabular}{ccc}
\hline Dielectric Material & Field Strength $(\mathrm{V} / \mathrm{mm})$ & $3^{\text {rd }}$ Harmonic Level $(\mathrm{dBm})$ \\
\hline Nylon-66 & 700 & -106 \\
Polythene & 2000 & -88 \\
Polystyrene & 2250 & -94 \\
Al.(AL $\left.\mathrm{O}_{2}\right)$ & 2250 & Not Detected \\
PTFE & 2250 & Not Detected \\
\hline
\end{tabular}




\section{Future Work}

The rest of the common used Dielectric materials (such as, PTFE, $\mathrm{AL}_{2} \mathrm{O}_{2}$, etc.) need to be tested by using different technique and/or different design for the measurement system to be able to detect the nonlinear behavior.

\section{Acknowledgments}

This project is funded by King Khalid University, Deanship of Scientific Research, project number (kku 151/2). I express my warm thanks to all academic staff of Deanship of Scientific Research for their support and guidance throughout project time.

\section{References}

[1] Khattab, T. and Rawlins, A.D. (1996) Principle of Low PIM Hardware Design. 13th National Radio Science Conference, NRSC'96, Cairo, 19-21 March 1996, 355-362. http://dx.doi.org/10.1109/NRSC.1996.551127

[2] Foord, A.P. and Rawlins, A.D. (1992) A Study of Passive Intermodulation Interference in Spce RF Hardware. ESA Report, University of Kent, Canterbury.

[3] Burfoot, J.C. and Taylor (1979) Polar Dielectrics and Their Applications. Macmillan Press, London.

[4] Landau, L.D. and Lifshitz, E.M. (1984) Electrodynamics of Continuous Media. Pregamon Press, Oxford.

[5] Stauss, G.H. (1980) Studies on the Reduction of Intrmodulation Generation in Communication Systems. NRL Memorandum Report 4233, 75-79.

[6] Smyth, C.P. (1995) Dielectric Behavior and Structure. McGraw-Hill, New York.

[7] Zheludev, I.S. (1971) Physics of Crystalline Dielectrcs, Volume 2, Electrical Properties. Plenum Press, New York.

[8] Bogoroditskii, N.P. and Pasynkov, V.V. (1968) Radio and Electronic Materials. Iliffe Books, London.

[9] Bottcher, C.J.F. (1973) Theory of Electric Polarization, Volume 1. Elsevier, Amsterdam, 318-323.

[10] Kumar, A. (1987) Passive IM Products Threaten High Power Satcom Systems. Microwave \& RF, 26, 98-103.

[11] Nishikawa, T., Ishikawa, Y. and Hattori, J. (1988) Measurement Method of Intermodulation Distortion of Dielectric Resonator. Japanese Journal of Applied Physics, 39-45. 
Scientific Research Publishing (SCIRP) is one of the largest Open Access journal publishers. It is currently publishing more than 200 open access, online, peer-reviewed journals covering a wide range of academic disciplines. SCIRP serves the worldwide academic communities and contributes to the progress and application of science with its publication.

Other selected journals from SCIRP are listed as below. Submit your manuscript to us via either submit@scirp.org or Online Submission Portal.
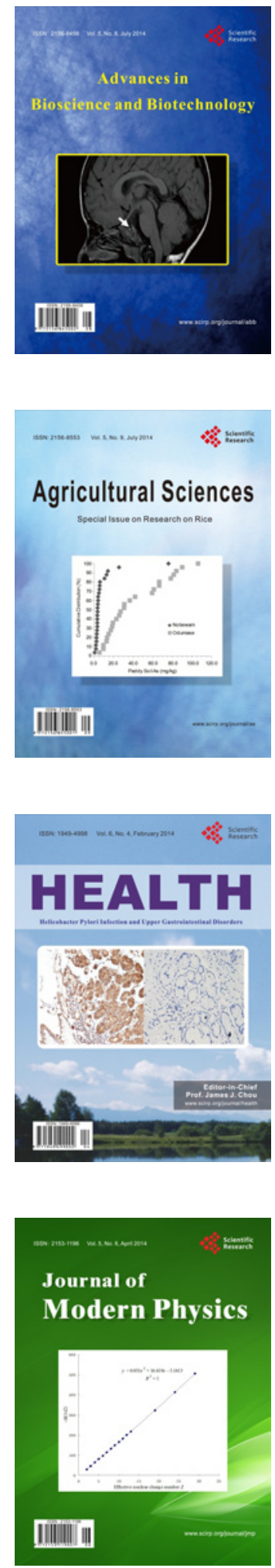
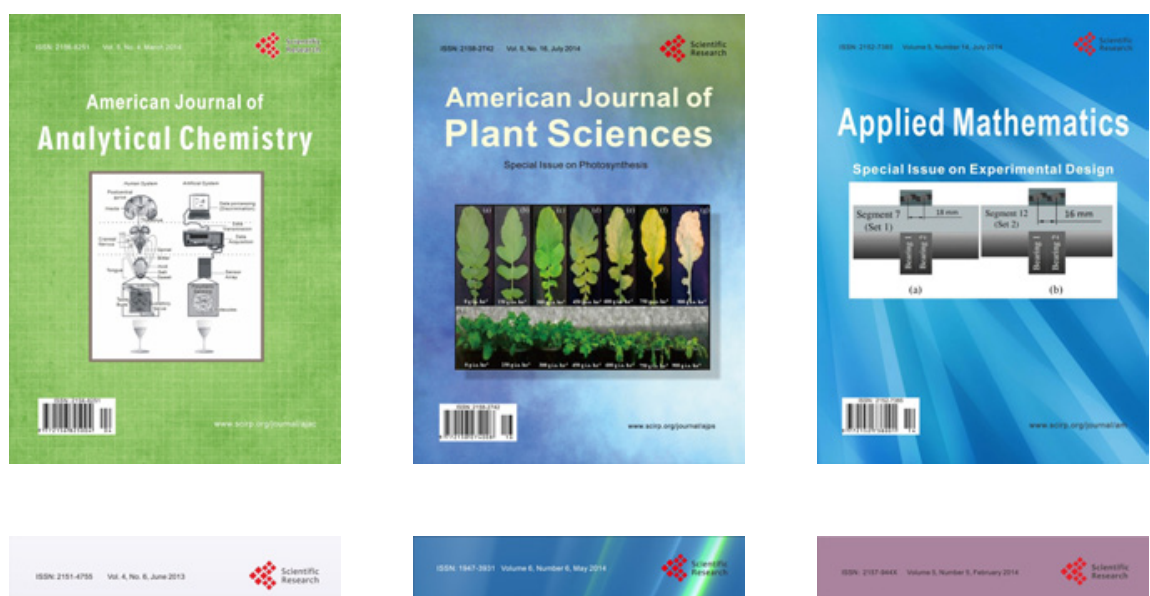

Creative Education
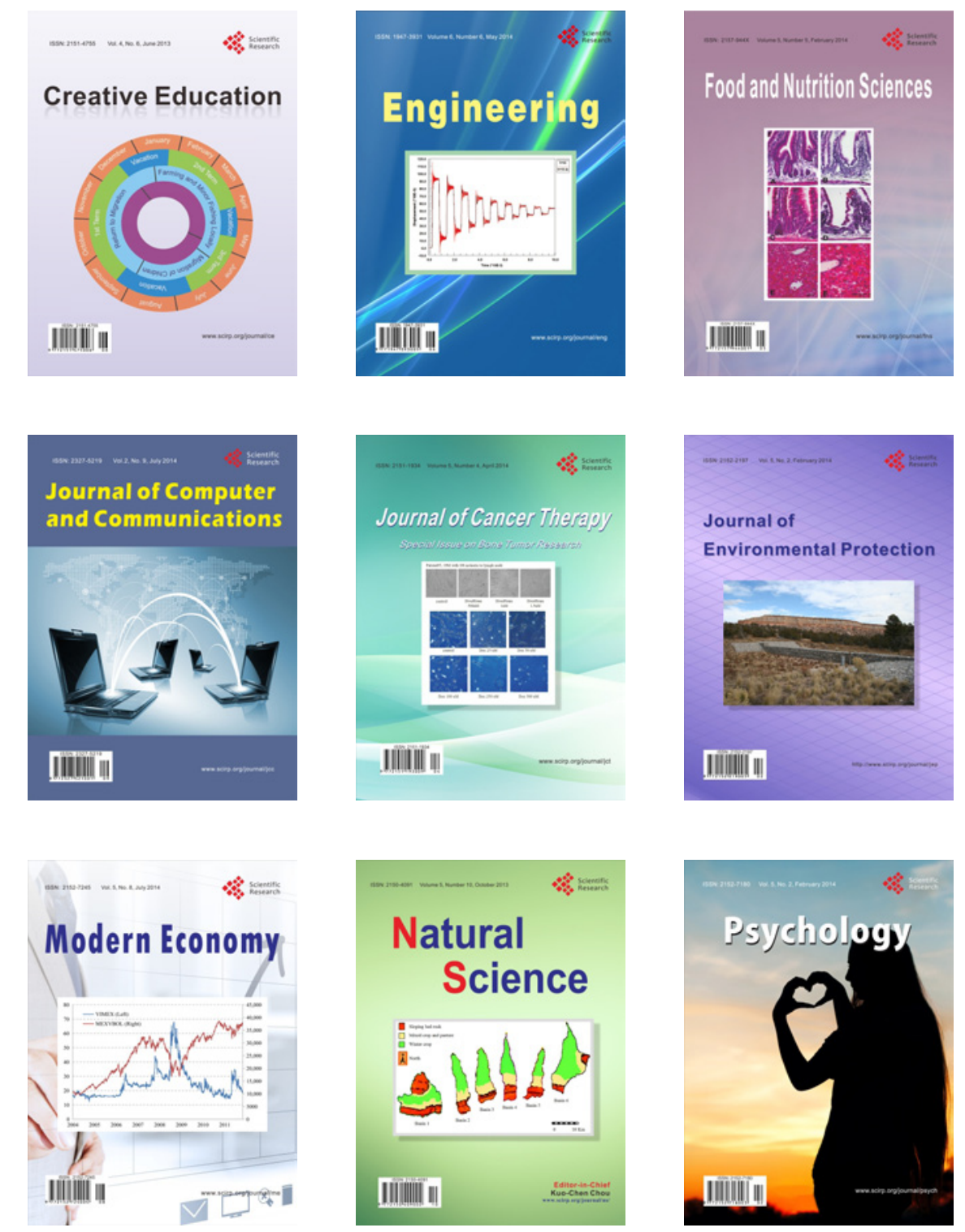